Original Article

\title{
INHIBITION OF UREASE ENZYME ACTIVITY BY UREA AND THIOUREA DERIVATIVES OF DIPEPTIDES CONJUGATED 2, 3-DICHLOROPHENYL PIPERAZINE
}

\author{
D. M. SUYOGA VARDHAN, H. K. KUMARA, H. PAVAN KUMAR, D. CHANNE GOWDA* \\ Department of Studies in Chemistry, University of Mysore, Manasagangotri, Mysore 570006, Karnataka, India \\ Email: dchannegowda@yahoo.co.in
}

Received: 24 Apr 2017 Revised and Accepted: 13 Jul 2017

\section{ABSTRACT}

Objective: Studies on enzyme inhibition remain an important area of pharmaceutical research since these studies have led to the discoveries of drugs used in a variety of physiological conditions. In search of novel urease enzyme inhibitors, four dipeptides were synthesized, conjugated to 2,3dichlorophenyl piperazine analogue and converted into urea/thiourea derivatives.

Methods: The peptides were synthesized by solution phase method and conjugated to 2,3-dichlorophenyl piperazine (PZN) using 1-ethyl-3-(3dimethyl aminopropyl) carbodiimide (EDCI)/hydroxybenzotriazole (HOBt) as a coupling agent and $N$-methylmorpholine (NMM) as a base. The tertbutyloxycarbonyl (Boc) group was removed using trifluoroacetic acid (TFA), neutralized with NMM and converted to urea and thiourea derivatives using substituted phenyl isocyanates and isothiocyanates respectively.

Results: Most of the synthesized analogues were found to be good inhibitors of urease enzyme activity. The conjugates of thiourea with $\mathrm{F}$ and $\mathrm{Cl}$ substituents at meta or para positions showed predominant urease inhibitory activity. The analogue 23 was nearly 10 fold ( $2 \mu \mathrm{M})$ more potent than the reference standard, $21.0 \pm 0.11 \mu \mathrm{M}$

Conclusion: The reported activity was correlated with some of the literature reported urease inhibitors and found that our compound 23 was more potent than the existing ones.

Keywords: Amino acids, Piperazine, Conjugation, Urea/thiourea, Urease inhibitors

(c) 2017 The Authors. Published by Innovare Academic Sciences Pvt Ltd. This is an open access article under the CC BY license (http://creativecommons.org/licenses/by/4.0/) DOI: http://dx.doi.org/10.22159/ijpps.2017v9i9.19425

\section{INTRODUCTION}

Activity of urease (E. C 3.5.1.5) has been shown to be an important virulence determinant in the pathogenesis of many clinical conditions, which are detrimental for human and animal health as well as for agriculture. Urease is directly involved in the formation of infectious stones and contributes to the pathogenesis of urolithiasis, pyelonephritis, ammonia and hepatic encephalopathy, hepatic coma, urinary catheter encrustation [1-3]. It is also known to be a major cause of pathologies induced by Helicobacter pylori (HP), which allows bacteria to survive at low $\mathrm{pH}$ of the stomach during colonization and therefore plays an important role in the pathogenesis of gastric and peptic ulcer [2]. In agriculture, high urease activity causes significant environmental and economic problems by releasing abnormally large amounts of ammonia into
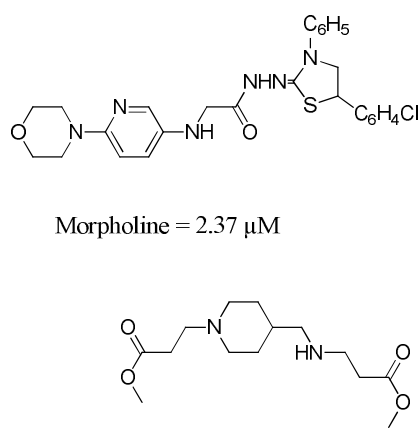

Substituted piperaidine $=19.38 \mu \mathrm{M}$

Urea is a common structural feature of many biologically active compounds such as enzyme inhibitors and pseudo peptides. The the atmosphere during urea fertilization. This further induces plant damage primarily by depriving them from their essential nutrient, and secondly ammonia toxicity and increase the $\mathrm{pH}$ of the soil $[1,2]$. Therefore, strategies based on urease inhibition were considered as the first line of treatment for infections caused by urease producing bacteria. Several classes of molecules have been tested as urease inhibitors (fig. 1) [4-9], but part of them was prevented from using in vivo because of their toxicity or instability. As a consequence agents currently used to inhibit urease have some deleterious side effects. Hence, the present work was intended to search for better compounds which would enable to replace the existing ones suffering from serious limitations. Meanwhile, the studies on novel urease inhibitors are essential not only for the basic research on urease biochemistry but also for the possible development of a highly needed therapy for urease mediated bacterial infections.

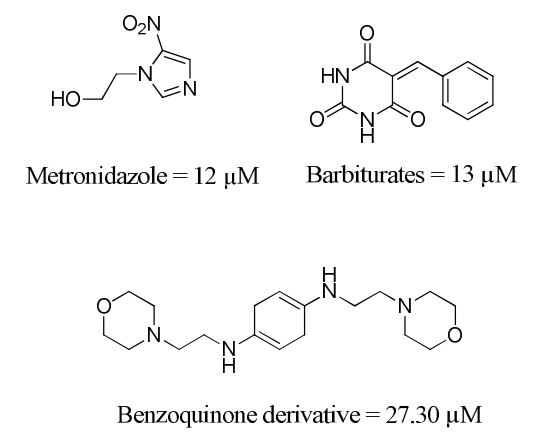

literature on urease inhibitors with possible applications in medicine showed urea derivatives and heterocyclic compounds 
constitute the major classes of structures with such activity. These ureas/thioureas display a wide range of biological activities including antibacterial, antifungal, antitubercular, antithyroid, anthelmintic, antidiabetic, anticancer, insecticidal, herbicidal and antimalarial properties [10-14].

Piperazine scaffold has been considered as a moiety possessing a myriad spectrum of medicinal properties. Diversity of biological responses profile has attracted the considerable interest of several researchers across the globe to explore this skeleton for its assorted therapeutic significance. Various novel classes of structurally different piperazine motifs have been designed and synthesized depicting potential interventions and versatile type of biological activities such as antiurease, antibacterial, antidiabetic, antiinflammatory, anticoagulant and also found to have used in acute toxicity study [15-19]. There is a large amount of literature dealing with the physiological significance of amino acids. Their effects on a variety of metabolic and physiological ramifications are among the most investigated topics in biomedical sciences. In addition, taking into consideration the need of more urease inhibitors and afore mentioned biological significance of piperazine, ureas/thioureas, amino acids functions and coupled with our research focused on the development of novel potential bioactive agents [20-23]. The aim of this report was to develop some new antiurease agents with better therapeutic potential.

\section{MATERIALS AND METHODS}

\section{General}

All amino acids used except glycine were of $L$-configuration unless otherwise mentioned. All Boc-amino acids, EDCI, HOBt and TFA were purchased from Advanced Chem. Tech. (Louisville, Kentucky, USA). Isobutyl chloroformate (IBCF) and NMM were purchased from Sigma Chemical Co. (St. Louis, MO). All solvents and reagents used for the synthesis were of analytical grade. The progress of the reaction was monitored by thin layer chromatography (TLC) using silica gel coated on glass plates with the solvent system comprising chloroform/methanol/acetic acid in the the ratio 98:2:3 $\left(\mathrm{R}_{\mathrm{f}}^{\mathrm{a}}\right)$ and 95:5:3 $\left(\mathrm{R}_{\mathrm{f}}^{\mathrm{b}}\right)$ throughout the study and the compounds on TLC plates were detected by iodine vapors. Melting points (MP) were determined on a Superfit melting point apparatus (India) and are uncorrected. Infrared (IR) spectra of the compounds were recorded on Jasco Spectrometer (USA). ${ }^{1} \mathrm{H}$ nuclear maganetic resonance (NMR) spectra were obtained on VARIAN $400 \mathrm{MHz}$ instrument (USA) using DMSO- $d_{6}$ and the chemical shifts are reported as parts per million $(\delta \mathrm{ppm})$ using TMS as an internal standard. Mass spectra were obtained on Bruker (model HP-1100) (USA) electrospray mass spectrometer. Elemental analysis was performed by using VARIO EL III Elementar (Germany). Jack bean urease enzyme for urease inhibition studies was purchased from Research Organics, Cleveland, while other chemicals were purchased from Sigma Aldrich.

\section{Synthesis}

\section{Synthesis of Boc-Xaa-Pro-OBzl where Xaa = Phe (I), Val (II),} $\operatorname{Tyr}\left(2,6-\mathrm{Cl}_{2} \mathrm{Bzl}\right)$ (III) and Lys(Z) (IV)

To a Boc-Xaa-OH (0.005 mol) dissolved in acetonitrile $(10 \mathrm{ml} / \mathrm{g})$ and cooled to $0{ }^{\circ} \mathrm{C}$ and then added NMM (1.10 ml, $\left.0.005 \mathrm{~mol}\right)$. The solution was cooled to $-15{ }^{\circ} \mathrm{C} \pm 1{ }^{\circ} \mathrm{C}$ and then IBCF $(0.77 \mathrm{ml}, 0.005$ mol) was added under stirring maintaining the temperature at-15 ${ }^{\circ} \mathrm{C}$. After stirring the reaction mixture for $10 \mathrm{~min}$ a precooled solution of HCl-Pro-OBzl (1.209 g) in DMF (12 ml) and NMM (1.09 $\mathrm{ml}, 0.005 \mathrm{~mol}$ ) was poured slowly. After $20 \mathrm{~min}$ the $\mathrm{pH}$ of the solution was adjusted to 8 by adding NMM and the reaction mixture was stirred overnight at room temperature.

Acetonitrile was removed under reduced pressure and the residual DMF solution was poured into about $200 \mathrm{ml}$ ice cold $90 \%$ saturated $\mathrm{KHCO}_{3}$ solution and stirred for $30 \mathrm{~min}$. The product precipitated was extracted into $\mathrm{CHCl}_{3}$. The organic layer was washed with $5 \%$ $\mathrm{NaHCO}_{3}$ solution $(3 \times 20 \mathrm{ml})$, water $(2 \times 20 \mathrm{ml})$ and $0.1 \mathrm{~N} \mathrm{HCl}$ solution $(3 \times 20 \mathrm{ml})$ followed by brine $(2 \times 20 \mathrm{ml})$. The chloroform layer was dried over anhydrous $\mathrm{Na}_{2} \mathrm{SO}_{4}$. The solvent was removed under reduced pressure and triturated with dry ether or petroleum ether to obtain the products I-IV.

\section{Synthesis of Boc-Xaa-Pro-OH}

Each dipeptide (I-IV) (0.004 mol) was dissolved in methanol (10 $\mathrm{ml} / \mathrm{g}$ ) and $1 \mathrm{~N} \mathrm{NaOH}(2 \mathrm{eq})$ was added and stirred for $2 \mathrm{~h}$ at $\mathrm{rt}$. The completion of the reaction was monitored by TLC and the solvent was evaporated, cooled, neutralized with cold $1 \mathrm{~N} \mathrm{HCl}$ and extracted with $\mathrm{CHCl}_{3}$. The organic layer was washed with $1 \mathrm{~N} \mathrm{HCl}$ followed by $\mathrm{H}_{2} \mathrm{O}$ and dried over anhydrous $\mathrm{Na}_{2} \mathrm{SO}_{4}$. The solvent was removed under pressure and triturated with ether, filtered, washed with ether and dried to obtain debenzylated peptides (V-VIII).

\section{Boc-Phe-Pro-OH (V)}

Yield $=92.0 \% ; \mathrm{R}_{\mathrm{f}}^{\mathrm{a}}=0.61 ; \mathrm{R}_{\mathrm{f}}^{\mathrm{b}}=0.75 ;$ M. P. $=97-100{ }^{\circ} \mathrm{C}$ (Lit. $100{ }^{\circ} \mathrm{C}$ ) [24]; ${ }^{1} \mathrm{H}$ NMR (DMSO- $d_{6}, \delta$ ppm): Boc $=1.41(9 \mathrm{H}, \mathrm{s}) ;$ Phe $=3.42-3.53$ $\left(2 \mathrm{H}, \mathrm{d},-\mathrm{-}^{\beta} \mathrm{CH}_{2}\right) ; 4.86-4.91\left(1 \mathrm{H}, \mathrm{m},-^{\alpha} \mathrm{CH}\right) ; 6.54-7.40(5 \mathrm{H}, \mathrm{m}, \mathrm{Ar}-\mathrm{H}) ;$ Pro $=$

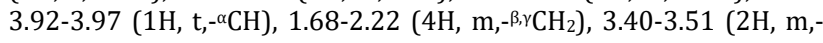
$\left.{ }^{\delta} \mathrm{CH}_{2}\right)$; Mass = $367.40\left(\mathrm{M}^{+}+1\right)$; Elem. Anal. = calcd. for $\mathrm{C}_{19} \mathrm{H}_{26} \mathrm{~N}_{2} \mathrm{O}_{5}$ : C: 62.97; H: 7.23; N: 7.73; Found: C: 62.95; H: 7.20; N: 7.73

\section{Boc-Val-Pro-OH (VI)}

Yield = 94.0\%; $\mathrm{R}_{\mathrm{f}}^{\mathrm{a}=}$ 0.56; $\mathrm{R}_{\mathrm{f}}^{\mathrm{b}}=0.68 ; \mathrm{M} . \mathrm{P} .=80-82{ }^{\circ} \mathrm{C}$ (Lit. $83^{\circ} \mathrm{C}$ ) [25]; ${ }^{1} \mathrm{H}$ NMR (DMSO- $\left.d_{6}, \delta \mathrm{ppm}\right):$ Boc $=1.42(9 \mathrm{H}, \mathrm{s}) ; \mathrm{Val}=1.03(6 \mathrm{H}, \mathrm{d}$,$\left.\left(\mathrm{CH}_{3}\right)_{2}\right), 1.88-1.90\left(1 \mathrm{H}, \mathrm{m},-{ }^{\beta} \mathrm{CH}\right), 3.53-3.64\left(1 \mathrm{H}, \mathrm{m},{ }^{-}{ }^{\alpha} \mathrm{CH}\right)$; Pro $=3.69-$ $3.73\left(1 \mathrm{H}, \mathrm{t},{ }^{\alpha} \mathrm{CH}\right), 1.90-2.35\left(4 \mathrm{H}, \mathrm{m},-{ }^{-\beta}, \mathrm{CH}_{2}\right), 2.97-3.52\left(2 \mathrm{H}, \mathrm{m},-^{-} \mathrm{CH}_{2}\right)$; Mass = $315.30\left(\mathrm{M}^{+}+1\right)$; Elem. Anal. = calcd. for $\mathrm{C}_{15} \mathrm{H}_{26} \mathrm{~N}_{2} \mathrm{O}_{5}$ : C: 57.31 ; H: 8.34; N: 8.91; Found: C: 57.32; H: 8.32; N: 8.88

\section{Boc-Tyr(2,6-Cl ${ }_{2}$ Bzl)-Pro-OH (VII)}

Yield = 89.2\%; $\mathrm{R}_{\mathrm{f}}^{\mathrm{a}}=0.56 ; \mathrm{R}_{\mathrm{f}}^{\mathrm{b}}=0.69 ;$ M. P. $=95-98{ }^{\circ} \mathrm{C} ;{ }^{1} \mathrm{H}$ NMR (DMSO- $d_{6}$, $\delta \mathrm{ppm}):$ Boc = $1.43(9 \mathrm{H}, \mathrm{s}) ; \mathrm{Tyr}=3.66-3.76\left(2 \mathrm{H}, \mathrm{d},-\mathrm{CH}_{2}\right) ; 4.81-4.87$ $\left(1 \mathrm{H}, \mathrm{t},{ }^{\alpha} \mathrm{CH}\right) ; 5.17\left(2 \mathrm{H}, \mathrm{s}\right.$, side chain- $\left.\mathrm{CH}_{2}\right) ; 6.70-7.90(7 \mathrm{H}, \mathrm{m}, \mathrm{ArH}) ;$ Pro = 3.35-3.48 (1H, t,- $\left.{ }^{\alpha} \mathrm{CH}\right), 1.68-1.88\left(4 \mathrm{H}, \mathrm{m},-{ }^{-\beta, \gamma} \mathrm{CH}_{2}\right), 2.91-3.13(2 \mathrm{H}, \mathrm{m}$,$\left.{ }^{\delta} \mathrm{CH}_{2}\right)$; Mass = $538.41\left(\mathrm{M}^{+}+1\right)$; Elem. Anal. = calcd. for $\mathrm{C}_{26} \mathrm{H}_{30} \mathrm{Cl}_{2} \mathrm{~N}_{2} \mathrm{O}_{6}: \mathrm{C}$ : 58.11; H: 5.63; N: 5.21; Found: C: 58.12; H: 5.62; N: 5.28

\section{Boc-Lys(Z)-Pro-OH (VIII)}

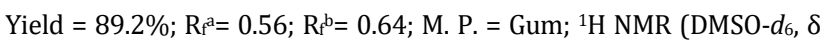
ppm): Boc = $1.43(9 \mathrm{H}, \mathrm{s}) ;$ Lys $=1.70-1.72\left(2 \mathrm{H}, \mathrm{m},{ }^{-}{ }^{8} \mathrm{CH}_{2}\right) ; 1.85-1.91(2 \mathrm{H}$, m, $\left.-\gamma \mathrm{CH}_{2}\right) ; 1.93-2.01\left(2 \mathrm{H}, \mathrm{m},-\mathrm{\beta CH}_{2}\right) ; 3.18-3.25\left(2 \mathrm{H}, \mathrm{m},-\mathrm{-CH}_{2}\right) ; 4.06(1 \mathrm{H}$, $\left.\mathrm{m},-^{-} \mathrm{CH}\right) ; 8.13(1 \mathrm{H}, \mathrm{s},-\mathrm{NH}) ; 5.15(2 \mathrm{H}, \mathrm{s}$, benzyl-CH$) ; 7.18-7.43(5 \mathrm{H}, \mathrm{m}$, $\mathrm{ArH}) ;$ Pro $=4.05-4.10\left(1 \mathrm{H}, \mathrm{t},-{ }^{\alpha} \mathrm{CH}\right), 1.67-2.25\left(4 \mathrm{H}, \mathrm{m},-{ }^{-\beta, \gamma} \mathrm{CH}_{2}\right), 3.72-3.78$ $\left(2 \mathrm{H}, \mathrm{m},-^{-} \mathrm{CH}_{2}\right) ;$ Mass $=478.51\left(\mathrm{M}^{+}+1\right) ;$ Elem. Anal. = calcd. for $\mathrm{C}_{24} \mathrm{H}_{35} \mathrm{~N}_{3} \mathrm{O}_{7}$ : C: 60.36; H: 7.39; N: 8.80; Found: C: 60.32; H: 7.32; N: 8.88

\section{General procedure for the coupling of piperazine derivative with peptides (1-4)}

PZN was synthesized as previously reported method [26]. To BocXaa-Pro-OH $(0.01 \mathrm{~mol})$ dissolved in acetonitrile $(10 \mathrm{ml} / \mathrm{g}$ of compound) and cooled to $0{ }^{\circ} \mathrm{C}$ was added NMM $(1.10 \mathrm{ml}, 0.01 \mathrm{~mol})$. To this EDCI (1.917 g, $0.01 \mathrm{~mol})$ was added and stirred while maintaining the temperature at $0{ }^{\circ} \mathrm{C}$. After stirring the reaction mixture for $10 \mathrm{~min}$ at this temperature, $\mathrm{HOBt}(1.531 \mathrm{~g}, 0.01 \mathrm{~mol})$ in dimethyl formamide (DMF) $(15 \mathrm{ml})$ was added slowly. The reaction mixture was stirred for an additional $10 \mathrm{~min}$ and a pre-cooled solution of PZN $(2.68 \mathrm{~g}, 0.01 \mathrm{~mol})$ and NMM $(1.10 \mathrm{ml}, 0.01 \mathrm{~mol})$ in DMF ( $25 \mathrm{ml}$ ) was added slowly. After $20 \mathrm{~min}, \mathrm{pH}$ of the solution was adjusted to 8 by the addition of NMM and the reaction mixture was stirred over night at room temperature. Acetonitrile was removed under reduced pressure and the residual DMF was poured into about $500 \mathrm{ml}$ ice-cold $90 \%$ saturated potassium bicarbonate $\left(\mathrm{KHCO}_{3}\right)$ solution and stirred for $30 \mathrm{~min}$. The precipitated compound was extracted into chloroform and washed sequentially with $5 \%$ sodium bicarbonate $\left(\mathrm{NaHCO}_{3}\right)$ solution $(3 \times 20 \mathrm{ml})$, water $(3 \times 20$ $\mathrm{ml}), 0.1 \mathrm{~N}$ cold $\mathrm{HCl}(3 \times 20 \mathrm{ml})$ followed by brine. The organic layer was dried over anhydrous $\mathrm{Na}_{2} \mathrm{SO}_{4}$, the solvent was removed under reduced pressure, triturated with ether, filtered and dried to get the conjugates 1-4.

tert-butyl (1-(2-(4-(2,3-dichlorophenyl)piperazine-1-carbonyl) pyrrolidin-1-yl)-1-oxo-3-phenylpropan-2-yl)carbamate (1)

Yield $=90.0 \% ; \mathrm{R}_{\mathrm{f}}^{\mathrm{a}}=0.58 ; \mathrm{R}_{\mathrm{f}}^{\mathrm{b}}=0.65 ; \mathrm{M} . \mathrm{P} .=$ Gum; ${ }^{1} \mathrm{H}$ NMR $\left(\right.$ DMSO- $d_{6}, \delta$ ppm): Boc = $1.43(9 \mathrm{H}, \mathrm{s})$; Phe = 3.41-3.51 $\left(2 \mathrm{H}, \mathrm{d},-\mathrm{-}^{-} \mathrm{CH}_{2}\right), 4.59-4.91$ $\left(1 \mathrm{H}, \mathrm{m},-^{-\alpha} \mathrm{CH}\right), 6.52-7.40(5 \mathrm{H}, \mathrm{m}, \mathrm{Ar}-\mathrm{H})$; Pro $=3.79-3.94\left(1 \mathrm{H}, \mathrm{t},{ }^{-\alpha} \mathrm{CH}\right)$, 
1.68-2.20 (4H, m, $\left.-\mathrm{-}_{\gamma} \mathrm{CH}_{2}\right), 3.38-3.49\left(2 \mathrm{H}, \mathrm{m},{ }^{-} \mathrm{CH}_{2}\right) ; \mathrm{PZN}=3.26-3.37$ (4H, m,- $\left.-\mathrm{CH}_{2}\right) ; 3.42-3.46\left(4 \mathrm{H}, \mathrm{m},-\mathrm{CH}_{2}\right) ; 6.52-7.40(3 \mathrm{H}, \mathrm{m}, \mathrm{Ar}-\mathrm{H})$. Mass $=576.48\left(\mathrm{M}^{+}+1\right)$; Elem. Anal. = calcd. for $\mathrm{C}_{29} \mathrm{H}_{36} \mathrm{~N}_{4} \mathrm{O}_{4}$ : C: 60.52; $\mathrm{H}$ : 6.30; N: 9.73; Found: C: 60.55; H: 6.31; N: 9.75

tert-butyl (1-(2-(4-(2,3-dichlorophenyl)piperazine-1-carbonyl) pyrrolidin-1-yl)-3-methyl-1-oxobutan-2-yl)carbamate (2)

Yield = 88.8\%; $\mathrm{R}_{\mathrm{f}}^{\mathrm{a}}=0.48 ; \mathrm{R}_{\mathrm{f}}^{\mathrm{b}}=0.57 ;$ M. P. $=$ Gum; ${ }^{1} \mathrm{H}$ NMR $\left(\right.$ DMSO- $d_{6}, \delta$ ppm): Boc = $1.43(9 \mathrm{H}, \mathrm{s}) ; \mathrm{Val}=1.06\left(6 \mathrm{H}, \mathrm{d},-\left(\mathrm{CH}_{3}\right)_{2}\right), 1.88-1.91(1 \mathrm{H}$, $\left.\mathrm{m},{ }^{\beta} \mathrm{CCH}\right), 4.34\left(1 \mathrm{H}, \mathrm{s},-^{-} \mathrm{CH}\right)$; Pro $=4.02\left(1 \mathrm{H}, \mathrm{t},{ }^{-\alpha} \mathrm{CH}\right), 1.68-2.24(4 \mathrm{H}, \mathrm{m},-$ $\left.\beta, \gamma \mathrm{CH}_{2}\right)$, 3.74-3.78 $\left(2 \mathrm{H}, \mathrm{m},{ }^{-} \mathrm{CH}_{2}\right) ; \mathrm{PZN}=3.34-3.42\left(4 \mathrm{H}, \mathrm{m},-\mathrm{CH}_{2}\right) ; 3.47-$ $3.50\left(4 \mathrm{H}, \mathrm{m},-\mathrm{CH}_{2}\right) ; 7.14-7.28(3 \mathrm{H}, \mathrm{m}, \mathrm{Ar}-\mathrm{H})$. Mass = $528.45\left(\mathrm{M}^{+}+1\right)$; Elem. Anal. = calcd. for $\mathrm{C}_{25} \mathrm{H}_{36} \mathrm{Cl}_{2} \mathrm{~N}_{4} \mathrm{O}_{4}$ : C: $56.92 ; \mathrm{H}: 6.88 ; \mathrm{N}: 10.62$; Found: C: 56.90; H: 6.82; N: 10.68

tert-butyl (3-(4-((2,6-dichlorobenzyl)oxy)phenyl)-1-(2-(4-(2,3dichlorophenyl)piperazine-1-carbonyl)pyrrolidin-1-yl)-1oxopropan-2-yl)carbamate (3)

Yield $=86.2 \% ; R_{\mathrm{f}}^{\mathrm{a}}=0.56 ; \mathrm{R}_{\mathrm{f}}^{\mathrm{b}}=0.68 ;$ M. P. $=$ Gum; ${ }^{1} \mathrm{H}$ NMR $\left(\right.$ DMSO- $d_{6}, \delta$ ppm): $\mathrm{Boc}=1.43(9 \mathrm{H}, \mathrm{s}) ; \mathrm{Tyr}=3.72\left(2 \mathrm{H}, \mathrm{d},{ }^{-} \mathrm{CH}_{2}\right) ; 4.81-4.89(1 \mathrm{H}, \mathrm{m},-$ $\left.{ }^{\alpha} \mathrm{CH}\right) ; 5.15$ (2H, s, side chain- $\left.\mathrm{CH}_{2}\right) ; 6.98-7.92(7 \mathrm{H}, \mathrm{m}, \mathrm{ArH}) ;$ Pro = 3.48 $\left(1 \mathrm{H}, \mathrm{t},{ }^{\alpha} \mathrm{CH}\right), 1.68-1.88\left(4 \mathrm{H}, \mathrm{m}_{-}-\beta, \gamma \mathrm{CH}_{2}\right), 2.94-3.16\left(2 \mathrm{H}, \mathrm{m}_{-}-{ }^{-} \mathrm{CH}_{2}\right) ; \mathrm{PZN}=$ 3.36-3.48 (4H, m, $\left.-\mathrm{CH}_{2}\right) ; 3.62-3.70\left(4 \mathrm{H}, \mathrm{m},-\mathrm{CH}_{2}\right) ; 6.98-7.92(3 \mathrm{H}, \mathrm{m}, \mathrm{Ar}-$ H). Mass = $751.52\left(\mathrm{M}^{+}+1\right)$; Elem. Anal. = calcd. for $\mathrm{C}_{36} \mathrm{H}_{40} \mathrm{Cl}_{4} \mathrm{~N}_{4} \mathrm{O}_{5}$ : C: 57.61; H: 5.37; N: 7.46; Found: C: 57.62; H: 5.32; N: 7.48

benzyl tert-butyl (6-(2-(4-(2,3-dichlorophenyl)piperazine-1carbonyl) pyrrolidin-1-yl)-6-oxohexane-1,5-diyl)dicarbamate (4)

Yield $=89.2 \% ; \mathrm{R}_{\mathrm{f}}^{\mathrm{a}}=0.56 ; \mathrm{R}_{\mathrm{f}}^{\mathrm{b}}=0.64 ;$ M. P. $=$ Gum; ${ }^{1} \mathrm{H}$ NMR $\left(\right.$ DMSO- $d_{6}, \delta$ ppm): Boc $=1.43(9 \mathrm{H}, \mathrm{s}) ;$ Lys $=1.70-1.72\left(2 \mathrm{H}, \mathrm{m},{ }^{-} \mathrm{CH}_{2}\right), 1.85-1.91$ $\left(2 \mathrm{H}, \mathrm{m},-\gamma \mathrm{CH}_{2}\right), 1.93-2.01\left(2 \mathrm{H}, \mathrm{m},-\beta \mathrm{CH}_{2}\right), 3.18-3.25\left(2 \mathrm{H}, \mathrm{m},-\in \mathrm{CH}_{2}\right) ; 4.06$ $\left(1 \mathrm{H}, \mathrm{m},{ }^{-} \mathrm{CH}\right) ; 8.13(1 \mathrm{H}, \mathrm{s},-\mathrm{NH}) ; 5.15\left(2 \mathrm{H}, \mathrm{s}\right.$, benzyl- $\left.\mathrm{CH}_{2}\right) ; 7.18-7.43$ $(5 \mathrm{H}, \mathrm{m}, \mathrm{ArH}) ;$ Pro $=4.05\left(1 \mathrm{H}, \mathrm{t},{ }^{\alpha} \mathrm{CH}\right), 1.65-2.21\left(4 \mathrm{H}, \mathrm{m},{ }^{-\beta}, \gamma \mathrm{CH}_{2}\right), 3.71-$ $3.76\left(2 \mathrm{H}, \mathrm{m}^{-}{ }^{-} \mathrm{CH}_{2}\right) ; \mathrm{PZN}=3.34-3.42\left(4 \mathrm{H}, \mathrm{m},-\mathrm{CH}_{2}\right) ; 3.47-3.50(4 \mathrm{H}, \mathrm{m},-$ $\left.\mathrm{CH}_{2}\right)$; 7.14-7.28 $(3 \mathrm{H}, \mathrm{m}, \mathrm{Ar}-\mathrm{H})$. Mass $=691.65\left(\mathrm{M}^{+}+1\right)$; Elem. Anal. $=$ calcd. for $\mathrm{C}_{34} \mathrm{H}_{45} \mathrm{Cl}_{2} \mathrm{~N}_{5} \mathrm{O}_{6}$ : C: 59.13; $\mathrm{H}: 6.57$; N: 10.14; Found: C: 59.12; H: $6.52 ; \mathrm{N}: 10.18$

General procedure for the synthesis of ureido and thioureido derivatives (5-36)

Each time, Boc-Xaa-Pro-PZN $(0.15 \mathrm{~g})$ was stirred with $1.5 \mathrm{ml}$ of TFA for $45 \mathrm{~min}$ at room temperature. After completion of the reaction (monitored by TLC), TFA was removed under vacuum, triturated with dry ether, filtered, washed with ether and dried to obtain TFA. H-Xaa-Pro-PZN.

Further, TFA. H-Xaa-Pro-PZN (0.001 mol) was dissolved in DMF (10 $\mathrm{ml} / \mathrm{g}$ of the compound), cooled to $0{ }^{\circ} \mathrm{C}$ and NMM $(0.10 \mathrm{ml}, 0.001$ mol) was added. To this solution respective substituted phenyl isocyanates and isothiocyanates $(0.0012 \mathrm{~mol})$ was added drop-wise while maintaining the temperature at $0{ }^{\circ} \mathrm{C}$. The reaction mixture was stirred for $8 \mathrm{~h}$ slowly warming to room temperature. DMF was evaporated under high vacuum and the residue was poured into about $50 \mathrm{ml}$ ice-cold $90 \%$ saturated $\mathrm{KHCO}_{3}$ solution and stirred for $30 \mathrm{~min}$. The precipitate was extracted into chloroform and washed sequentially with $5 \% \mathrm{NaHCO}_{3}$ solution $(2 \times 10 \mathrm{ml})$, water $(2 \times 10$ $\mathrm{ml}), 0.1 \mathrm{~N}$ citric acid $(2 \times 10 \mathrm{ml})$ followed by brine. The organic layer was dried over anhydrous $\mathrm{Na}_{2} \mathrm{SO}_{4}$. The solvent was removed under reduced pressure, triturated with hexane filtered and dried under vacuum to obtain urea/thiourea derivatives (5-36).

\section{Pharmacology}

\section{Anti-urease assay (in vitro)}

Reaction mixtures comprising $25 \mu$ of jack bean urease enzyme ( 10 $\mathrm{mg} / \mathrm{ml}$ ) solution and $55 \mu \mathrm{l}$ of buffers containing $100 \mathrm{mmol}$ urea were incubated with $5 \mu \mathrm{l}$ of the test compounds $0.5-500 \mathrm{mmol}$ concentration) at $30^{\circ} \mathrm{C}$ for $15 \mathrm{~min}$ in 96 -well plates. Urease activity was determined by measuring ammonia production using the indophenols method as described by Weatherburn [27]. Breifly, 45 $\mu \mathrm{l}$ each of phenol reagent $(1 \% \mathrm{w} / \mathrm{v}$ phenol and $0.005 \% \mathrm{w} / \mathrm{v}$ sodium nitroprusside) and $70 \mu \mathrm{l}$ of alkali reagent $(0.5 \% \mathrm{w} / \mathrm{v} \mathrm{NaOH}$ and $0.1 \%$ active chloride $\mathrm{NaOCl}$ ) were added to each well. The increasing absorbance at $630 \mathrm{~nm}$ was measured after $50 \mathrm{~min}$ using a microplate reader (Multimode plate reader, VARIOSKAN, Germany). All the reactions were performed in triplicate in a final volume of $200 \mu$. The entire assays were performed at pH 6.8 [28]. Percentage inhibition was calculated from the formula:

$$
\% \text { Inhibition }=100-\frac{\text { OD testwell }}{\text { OD control }} \times 100
$$

\section{RESULTS AND DISCUSSION}

\section{Chemistry}

In the present study, we have synthesized proline containing dipeptides and conjugated to 2,3-dichlorophenyl piperazine. These dipeptide-heterocyclic conjugates were further derivatized into ureas and thioureas by reacting with substituted phenyl isocyanates/isothiocyanates (Scheme).

Yields of the compounds were found to be>85\% and were characterized by IR, ${ }^{1} \mathrm{H}$ NMR, mass spectroscopic techniques. IR spectra of ureas and thioureas exhibited peaks at $v \sim 1620 \mathrm{~cm}^{-1}$ and $\sim 2040 \mathrm{~cm}^{-1}$ for $\mathrm{C}=0$ and $\mathrm{C}=\mathrm{S}$ respectively. ${ }^{1} \mathrm{H}$ NMR spectra showed singlet for-NH at $\delta \sim 8.75$ and multiples for another-NH proton at $\delta$ $\sim 8.31$ for urea derivatives. On the other hand, $\delta$ singlet at $\sim 9.70$ for-NH and multiplet at $\delta \sim 8.49$ for $\mathrm{NH}$ proton was observed for thiourea derivatives. Further, all other peaks were exactly matching the structure. The physical and mass; IR and NMR data of all the synthesized compounds are presented in table 1 and 2 respectively.

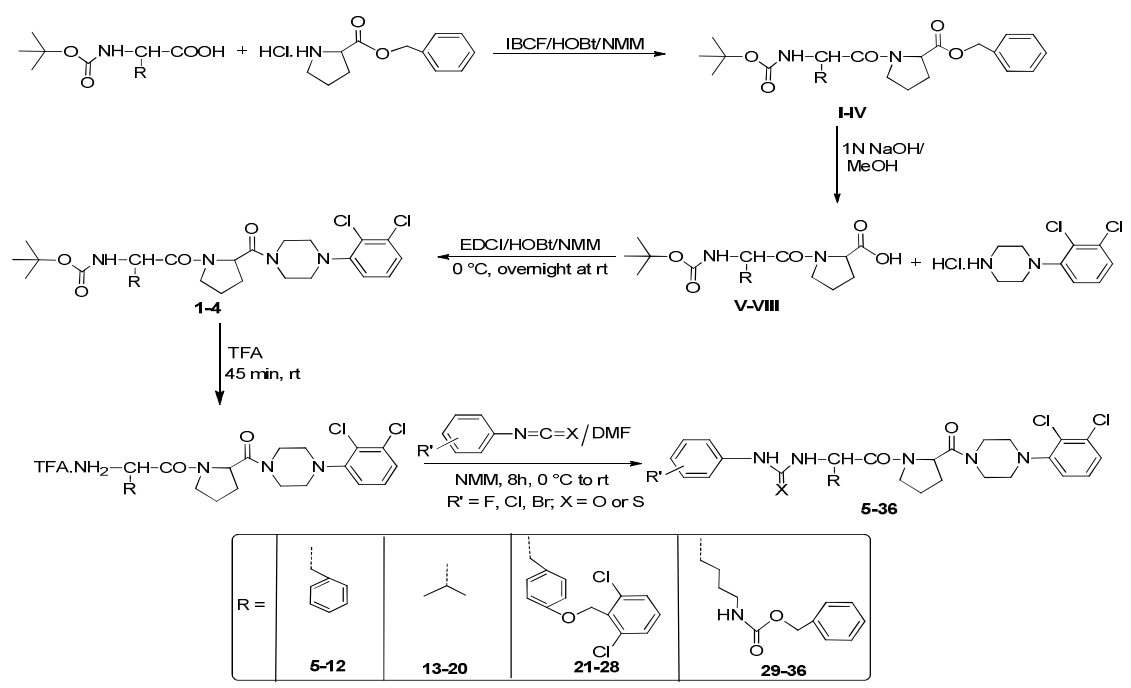

Scheme: Synthesis of ureido and thioureido derivatives of peptide conjugated heterocycle 
Table 1: Physical and mass data of synthesized urea/thiourea derivatives (5-36)

\begin{tabular}{|c|c|c|c|c|c|c|c|c|}
\hline Entry & $\mathbf{R}$ & $\mathbf{X}$ & $\mathbf{R}_{\mathbf{f}^{\mathbf{a}}}$ & $\mathbf{R}_{\mathbf{f}}^{\mathbf{b}}$ & Yield (\%) & MP $\left({ }^{\circ} \mathrm{C}\right)$ & Mol. For. & Mass $\left(M^{+}+1\right)$ \\
\hline 5 & $3 \mathrm{~F}$ & 0 & 0.53 & 0.61 & 91 & 75 & $\mathrm{C}_{31} \mathrm{H}_{32} \mathrm{Cl}_{2} \mathrm{FN}_{5} \mathrm{O}_{3}$ & 613.50 \\
\hline 6 & $4 \mathrm{~F}$ & 0 & 0.55 & 0.63 & 88 & 73 & $\mathrm{C}_{31} \mathrm{H}_{32} \mathrm{Cl}_{2} \mathrm{FN}_{5} \mathrm{O}_{3}$ & 613.54 \\
\hline 7 & $4 \mathrm{~F}$ & $\mathrm{~S}$ & 0.51 & 0.59 & 94 & 58 & $\mathrm{C}_{31} \mathrm{H}_{32} \mathrm{Cl}_{2} \mathrm{FN}_{5} \mathrm{O}_{2} \mathrm{~S}$ & 629.55 \\
\hline 8 & $3 \mathrm{Cl}$ & 0 & 0.49 & 0.58 & 85 & 72 & $\mathrm{C}_{31} \mathrm{H}_{32} \mathrm{Cl}_{3} \mathrm{~N}_{5} \mathrm{O}_{3}$ & 629.10 \\
\hline 9 & $3 \mathrm{Cl}$ & $\mathrm{S}$ & 0.45 & 0.56 & 84 & 58 & $\mathrm{C}_{31} \mathrm{H}_{32} \mathrm{Cl}_{3} \mathrm{~N}_{5} \mathrm{O}_{2} \mathrm{~S}$ & 646.10 \\
\hline 10 & $4 \mathrm{Cl}$ & $\mathrm{S}$ & 0.42 & 0.57 & 86 & $60-62$ & $\mathrm{C}_{31} \mathrm{H}_{32} \mathrm{Cl}_{3} \mathrm{~N}_{5} \mathrm{O}_{2} \mathrm{~S}$ & 646.12 \\
\hline 11 & $3 \mathrm{Br}$ & 0 & 0.52 & 0.65 & 88 & 68 & $\mathrm{C}_{31} \mathrm{H}_{32} \mathrm{BrCl}_{2} \mathrm{~N}_{5} \mathrm{O}_{3}$ & $\begin{array}{l}674.12 \\
676.15\end{array}$ \\
\hline 12 & $3 \mathrm{Br}$ & S & 0.50 & 0.62 & 90 & 55 & $\mathrm{C}_{31} \mathrm{H}_{32} \mathrm{BrCl}_{2} \mathrm{~N}_{5} \mathrm{O}_{3}$ & $\begin{array}{l}690.02 \\
692.09\end{array}$ \\
\hline 13 & $3 F$ & 0 & 0.49 & 0.58 & 92 & 58 & $\mathrm{C}_{27} \mathrm{H}_{32} \mathrm{Cl}_{2} \mathrm{FN}_{5} \mathrm{O}_{3}$ & 565.45 \\
\hline 14 & $4 \mathrm{~F}$ & 0 & 0.45 & 0.56 & 83 & $58-60$ & $\mathrm{C}_{27} \mathrm{H}_{32} \mathrm{Cl}_{2} \mathrm{FN}_{5} \mathrm{O}_{3}$ & 565.52 \\
\hline 15 & $4 \mathrm{~F}$ & $\mathrm{~S}$ & 0.43 & 0.53 & 86 & Gum & $\mathrm{C}_{27} \mathrm{H}_{32} \mathrm{Cl}_{2} \mathrm{FN}_{5} \mathrm{O}_{2} \mathrm{~S}$ & 581.58 \\
\hline 16 & $3 \mathrm{Cl}$ & 0 & 0.47 & 0.58 & 81 & Gum & $\mathrm{C}_{27} \mathrm{H}_{32} \mathrm{Cl}_{3} \mathrm{~N}_{5} \mathrm{O}_{3}$ & 581.90 \\
\hline 17 & $3 \mathrm{Cl}$ & $\mathrm{S}$ & 0.45 & 0.55 & 84 & Gum & $\mathrm{C}_{27} \mathrm{H}_{32} \mathrm{Cl}_{3} \mathrm{~N}_{5} \mathrm{O}_{2} \mathrm{~S}$ & 598.02 \\
\hline 18 & $4 \mathrm{Cl}$ & $\mathrm{S}$ & 0.44 & 0.55 & 88 & Gum & $\mathrm{C}_{27} \mathrm{H}_{32} \mathrm{Cl}_{3} \mathrm{~N}_{5} \mathrm{O}_{2} \mathrm{~S}$ & 598.05 \\
\hline 19 & $3 \mathrm{Br}$ & 0 & 0.46 & 0.59 & 90 & 55 & $\mathrm{C}_{27} \mathrm{H}_{32} \mathrm{BrCl}_{2} \mathrm{~N}_{5} \mathrm{O}_{3}$ & $\begin{array}{l}625.35 \\
627.42\end{array}$ \\
\hline 20 & $3 \mathrm{Br}$ & S & 0.44 & 0.57 & 91 & Gum & $\mathrm{C}_{27} \mathrm{H}_{32} \mathrm{BrCl}_{2} \mathrm{~N}_{5} \mathrm{O}_{2} \mathrm{~S}$ & $\begin{array}{l}641.50 \\
643.38\end{array}$ \\
\hline 21 & $3 \mathrm{~F}$ & 0 & 0.60 & 0.71 & 92 & $85-87$ & $\mathrm{C}_{38} \mathrm{H}_{36} \mathrm{Cl}_{4} \mathrm{FN}_{5} \mathrm{O}_{4}$ & 788.50 \\
\hline 22 & $4 \mathrm{~F}$ & 0 & 0.63 & 0.70 & 90 & 90 & $\mathrm{C}_{38} \mathrm{H}_{36} \mathrm{Cl}_{4} \mathrm{FN}_{5} \mathrm{O}_{4}$ & 788.48 \\
\hline 23 & $4 \mathrm{~F}$ & $\mathrm{~S}$ & 0.58 & 0.66 & 95 & $75-77$ & $\mathrm{C}_{38} \mathrm{H}_{36} \mathrm{Cl}_{4} \mathrm{FN}_{5} \mathrm{O}_{3} \mathrm{~S}$ & 804.63 \\
\hline 24 & $3 \mathrm{Cl}$ & 0 & 0.63 & 0.71 & 88 & 65 & $\mathrm{C}_{38} \mathrm{H}_{36} \mathrm{Cl}_{5} \mathrm{~N}_{5} \mathrm{O}_{4}$ & 804.85 \\
\hline 25 & $3 \mathrm{Cl}$ & S & 0.59 & 0.68 & 89 & $62-64$ & $\mathrm{C}_{38} \mathrm{H}_{36} \mathrm{Cl}_{5} \mathrm{~N}_{5} \mathrm{O}_{3} \mathrm{~S}$ & 821.09 \\
\hline 26 & $4 \mathrm{Cl}$ & $\mathrm{S}$ & 0.57 & 0.65 & 86 & 65 & $\mathrm{C}_{38} \mathrm{H}_{36} \mathrm{Cl}_{5} \mathrm{~N}_{5} \mathrm{O}_{3} \mathrm{~S}$ & 821.12 \\
\hline 27 & $3 \mathrm{Br}$ & 0 & 0.61 & 0.67 & 88 & 82 & $\mathrm{C}_{38} \mathrm{H}_{36} \mathrm{BrCl}_{4} \mathrm{~N}_{5} \mathrm{O}_{4}$ & $\begin{array}{l}849.51 \\
851.21\end{array}$ \\
\hline 28 & $3 \mathrm{Br}$ & S & 0.57 & 0.63 & 90 & 70 & $\mathrm{C}_{38} \mathrm{H}_{36} \mathrm{BrCl}_{4} \mathrm{~N}_{5} \mathrm{O}_{3} \mathrm{~S}$ & $\begin{array}{l}865.58 \\
867.61\end{array}$ \\
\hline 29 & $3 F$ & 0 & 0.49 & 0.57 & 80 & 72 & $\mathrm{C}_{36} \mathrm{H}_{41} \mathrm{Cl}_{2} \mathrm{FN}_{6} \mathrm{O}_{5}$ & 728.63 \\
\hline 30 & $4 \mathrm{~F}$ & 0 & 0.45 & 0.59 & 84 & 75 & $\mathrm{C}_{36} \mathrm{H}_{41} \mathrm{Cl}_{2} \mathrm{FN}_{6} \mathrm{O}_{5}$ & 727.71 \\
\hline 31 & $4 \mathrm{~F}$ & $\mathrm{~S}$ & 0.42 & 0.55 & 86 & $60-62$ & $\mathrm{C}_{36} \mathrm{H}_{41} \mathrm{Cl}_{2} \mathrm{FN}_{6} \mathrm{O}_{4} \mathrm{~S}$ & 744.75 \\
\hline 32 & $3 \mathrm{Cl}$ & 0 & 0.44 & 0.58 & 81 & 65 & $\mathrm{C}_{36} \mathrm{H}_{41} \mathrm{Cl}_{3} \mathrm{~N}_{6} \mathrm{O}_{5}$ & 745.12 \\
\hline 33 & $3 \mathrm{Cl}$ & $\mathrm{S}$ & 0.45 & 0.55 & 87 & 58 & $\mathrm{C}_{36} \mathrm{H}_{41} \mathrm{Cl}_{3} \mathrm{~N}_{6} \mathrm{O}_{4} \mathrm{~S}$ & 761.17 \\
\hline 34 & $4 \mathrm{Cl}$ & $\mathrm{S}$ & 0.47 & 0.56 & 84 & $55-57$ & $\mathrm{C}_{36} \mathrm{H}_{41} \mathrm{Cl}_{3} \mathrm{~N}_{6} \mathrm{O}_{4} \mathrm{~S}$ & 761.20 \\
\hline 35 & $3 \mathrm{Br}$ & 0 & 0.49 & 0.58 & 82 & $56-58$ & $\mathrm{C}_{36} \mathrm{H}_{41} \mathrm{BrCl}_{2} \mathrm{~N}_{6} \mathrm{O}_{5}$ & $\begin{array}{l}789.58 \\
791.12\end{array}$ \\
\hline 36 & $3 \mathrm{Br}$ & S & 0.41 & 0.53 & 90 & 54 & $\mathrm{C}_{36} \mathrm{H}_{41} \mathrm{Cl}_{2} \mathrm{~N}_{6} \mathrm{O}_{4} \mathrm{~S}$ & $\begin{array}{l}805.58 \\
807.44\end{array}$ \\
\hline
\end{tabular}

$\mathrm{R}_{\mathrm{f}}=$ Retention factor, MP= Melting point, $\mathrm{b}=$ The chemical shift values for the fragment 'Heterocycle' of the compounds $\mathbf{5}-\mathbf{3 6}$ are almost same as obtained for compound 5

Table 2: IR and NMR values of urea and thiourea derivatives of dipeptides conjugated PZN

\begin{tabular}{|c|c|c|c|c|c|}
\hline \multirow[t]{2}{*}{ Entry } & \multirow[t]{2}{*}{$\mathbf{R} / \mathbf{X}$} & \multicolumn{3}{|c|}{ IR v, $\mathbf{c m}^{-1}$} & \multirow[t]{2}{*}{${ }^{1} \mathrm{H}$ NMR (DMSO $d_{6}, \delta$ ppm) } \\
\hline & & CO & CS & NH & \\
\hline 5 & $3 \mathrm{~F}(0)$ & 1622 & & 3328 & $\begin{array}{l}\text { Urea = 8.24 }(1 \mathrm{H}, \mathrm{s},-\mathrm{NH}), 6.54-7.42(4 \mathrm{H}, \mathrm{m}, \mathrm{Ar}-\mathrm{H}) ; \\
\text { Phe = } 7.93(1 \mathrm{H}, \mathrm{s},-\mathrm{NH}), 6.54-7.44(5 \mathrm{H}, \mathrm{m}, \mathrm{Ar}-\mathrm{H}), 4.59-4.92\left(1 \mathrm{H}, \mathrm{m},{ }^{\alpha} \mathrm{CH}\right), 3.42-3.56\left(2 \mathrm{H}, \mathrm{d},{ }^{\beta} \mathrm{CH}_{2}\right) ; \\
\text { Pro = 3.86-3.94 }\left(1 \mathrm{H}, \mathrm{t},{ }^{\alpha} \mathrm{CH}\right), 1.68-2.18\left(4 \mathrm{H}, \mathrm{m},{ }^{-\beta, \gamma} \mathrm{CH}_{2}\right), 3.41-3.52\left(2 \mathrm{H}, \mathrm{t},{ }^{-} \mathrm{CH}_{2}\right) \\
\text { Heterocycle }^{\mathrm{b}}=3.26-3.37\left(4 \mathrm{H}, \mathrm{m},-\mathrm{CH}_{2}\right) ; 3.41-3.45\left(4 \mathrm{H}, \mathrm{m},-\mathrm{CH}_{2}\right) ; 6.54-7.44(3 \mathrm{H}, \mathrm{m}, \mathrm{Ar}-\mathrm{H}) .\end{array}$ \\
\hline 6 & $4 \mathrm{~F}(0)$ & 1624 & & 3324 & $\begin{array}{l}\text { Urea }=8.19(1 \mathrm{H}, \mathrm{s},-\mathrm{NH}), 6.52-7.41(4 \mathrm{H}, \mathrm{m}, \mathrm{Ar}-\mathrm{H}) ; \\
\text { Phe = } 7.91(1 \mathrm{H}, \mathrm{s},-\mathrm{NH}) ; 6.66-7.58(5 \mathrm{H}, \mathrm{m}, \mathrm{Ar}-\mathrm{H}), 4.65-4.84\left(1 \mathrm{H}, \mathrm{m},{ }^{\alpha} \mathrm{CH}\right), 3.56-3.66\left(2 \mathrm{H}, \mathrm{d},{ }^{\beta} \mathrm{CH}_{2}\right) ; \\
\text { Pro }=3.82-3.91\left(1 \mathrm{H}, \mathrm{t},{ }^{\alpha} \mathrm{CH}\right), 1.64-2.28\left(4 \mathrm{H}, \mathrm{m},{ }^{-\beta, \gamma} \mathrm{CH}_{2}\right), 3.38-3.50\left(2 \mathrm{H}, \mathrm{t},{ }^{-} \mathrm{CH}_{2}\right)\end{array}$ \\
\hline 7 & $4 \mathrm{~F}(\mathrm{~S})$ & 1620 & 2125 & 3325 & $\begin{array}{l}\text { Thiourea = } 8.79(1 \mathrm{H}, \mathrm{s},-\mathrm{NH}), 6.52-7.43(4 \mathrm{H}, \mathrm{m}, \mathrm{Ar}-\mathrm{H}) ; \\
\text { Phe }=7.93(1 \mathrm{H}, \mathrm{s}, \mathrm{NH}), 6.74-7.63(5 \mathrm{H}, \mathrm{m}, \mathrm{Ar}-\mathrm{H}), 4.83-4.89\left(1 \mathrm{H}, \mathrm{m},-^{\alpha} \mathrm{CH}\right), 3.57-3.70\left(2 \mathrm{H}, \mathrm{d},{ }^{\beta} \mathrm{CH}_{2}\right) \\
\text { Pro }=3.79-3.94\left(1 \mathrm{H}, \mathrm{t},{ }^{\alpha} \mathrm{CH}\right), 3.38-3.48\left(2 \mathrm{H}, \mathrm{t},{ }^{-}{ }^{-} \mathrm{CH}_{2}\right), 1.68-2.26\left(4 \mathrm{H}, \mathrm{m},{ }^{\beta}, \mathrm{CH}_{2}\right)\end{array}$ \\
\hline 8 & $3 \mathrm{Cl}(0)$ & 1618 & & 3319 & $\begin{array}{l}\text { Urea }=8.29(1 \mathrm{H}, \mathrm{s},-\mathrm{NH}), 6.50-7.38(4 \mathrm{H}, \mathrm{m}, \mathrm{Ar}-\mathrm{H}) ; \\
\text { Phe = } 7.96(1 \mathrm{H}, \mathrm{s}, \mathrm{NH}) ; 6.54-7.48(5 \mathrm{H}, \mathrm{m}, \mathrm{Ar}-\mathrm{H}), 4.52-4.84\left(1 \mathrm{H}, \mathrm{m},{ }^{-}{ }^{\alpha} \mathrm{CH}\right), 3.41-3.56\left(2 \mathrm{H}, \mathrm{d},{ }^{\beta} \mathrm{CH}_{2}\right) \\
\text { Pro }=3.79-3.94\left(1 \mathrm{H}, \mathrm{t},{ }^{\alpha} \mathrm{CH}\right), 1.62-2.28\left(4 \mathrm{H}, \mathrm{m},{ }^{-\beta, \gamma} \mathrm{CH}_{2}\right), 3.41-3.52\left(2 \mathrm{H}, \mathrm{t},{ }^{-} \mathrm{CH}_{2}\right)\end{array}$ \\
\hline 9 & $3 \mathrm{Cl}(\mathrm{S})$ & 1625 & 2132 & 3328 & $\begin{array}{l}\text { Thiourea = } 8.82(1 \mathrm{H}, \mathrm{s},-\mathrm{NH}), 6.55-7.48(4 \mathrm{H}, \mathrm{m}, \mathrm{Ar}-\mathrm{H}) ; \\
\text { Phe }=7.95(1 \mathrm{H}, \mathrm{s}, \mathrm{NH}), 6.55-7.48(5 \mathrm{H}, \mathrm{m}, \mathrm{Ar}-\mathrm{H}), 4.55-4.83\left(1 \mathrm{H}, \mathrm{m},-^{-\alpha} \mathrm{CH}\right), 3.41-3.50\left(2 \mathrm{H}, \mathrm{d},{ }^{\beta} \mathrm{CH}_{2}\right) \\
\text { Pro }=3.74-3.95\left(1 \mathrm{H}, \mathrm{t},{ }^{\alpha} \mathrm{CH}\right), 3.43-3.52\left(2 \mathrm{H}, \mathrm{t},{ }^{-}{ }^{-} \mathrm{CH}_{2}\right), 1.68-2.16\left(4 \mathrm{H}, \mathrm{m},{ }^{\beta}, \gamma \mathrm{CH}_{2}\right)\end{array}$ \\
\hline 10 & $4 \mathrm{Cl}(\mathrm{S})$ & 1624 & 2127 & 3320 & $\begin{array}{l}\text { Thiourea = } 8.74(1 \mathrm{H}, \mathrm{s},-\mathrm{NH}), 6.52-7.43(4 \mathrm{H}, \mathrm{m}, \mathrm{Ar}-\mathrm{H}) ; \\
\text { Phe }=7.89(1 \mathrm{H}, \mathrm{s}, \mathrm{NH}), 6.52-7.43(5 \mathrm{H}, \mathrm{m}, \mathrm{Ar}-\mathrm{H}), 4.53-4.91\left(1 \mathrm{H}, \mathrm{m},-^{-\alpha} \mathrm{CH}\right), 3.41-3.51\left(2 \mathrm{H}, \mathrm{d},{ }^{\beta} \mathrm{CH}_{2}\right) ; \\
\text { Pro }=3.79-3.92\left(1 \mathrm{H}, \mathrm{t},{ }^{\alpha} \mathrm{CH}\right), 1.64-2.12\left(4 \mathrm{H}, \mathrm{m},-{ }^{-\beta, \gamma} \mathrm{CH}_{2}\right), 3.39-3.46\left(2 \mathrm{H}, \mathrm{t},{ }^{-}{ }^{-} \mathrm{CH}_{2}\right)\end{array}$ \\
\hline 11 & $3 \mathrm{Br}(0)$ & 1620 & & 3325 & $\begin{array}{l}\text { Urea = } 8.27(1 \mathrm{H}, \mathrm{s},-\mathrm{NH}), 6.56-7.48(4 \mathrm{H}, \mathrm{m}, \mathrm{Ar}-\mathrm{H}) \\
\text { Phe }=7.92(1 \mathrm{H}, \mathrm{s}, \mathrm{NH}), 6.56-7.48(5 \mathrm{H}, \mathrm{m}, \mathrm{Ar}-\mathrm{H}), 4.50-4.94\left(1 \mathrm{H}, \mathrm{m},-^{-\alpha} \mathrm{CH}\right), 3.46-3.56\left(2 \mathrm{H}, \mathrm{d},{ }^{\beta} \mathrm{CH}_{2}\right) \\
\text { Pro }=3.74-3.92\left(1 \mathrm{H}, \mathrm{t},{ }^{\alpha} \mathrm{CH}\right), 3.41-3.50\left(2 \mathrm{H}, \mathrm{t},{ }^{-}{ }^{-} \mathrm{CH}_{2}\right), 1.68-2.18\left(4 \mathrm{H}, \mathrm{m},{ }^{-\beta}, \mathrm{CH}_{2}\right)\end{array}$ \\
\hline 12 & $3 \mathrm{Br}(\mathrm{S})$ & 1624 & 2123 & 3324 & $\begin{array}{l}\text { Thiourea = } 8.66(1 \mathrm{H}, \mathrm{s},-\mathrm{NH}), 6.58-7.43(4 \mathrm{H}, \mathrm{m}, \mathrm{Ar}-\mathrm{H}) \\
\text { Phe }=7.92(1 \mathrm{H}, \mathrm{s}, \mathrm{NH}), 4.53-4.83\left(1 \mathrm{H}, \mathrm{m},{ }^{-}{ }^{\alpha} \mathrm{CH}\right), 3.41-3.50\left(2 \mathrm{H}, \mathrm{d},{ }^{\beta} \mathrm{CH}_{2}\right), 6.58-7.43(5 \mathrm{H}, \mathrm{m}, \mathrm{Ar}-\mathrm{H})\end{array}$ \\
\hline
\end{tabular}




\begin{tabular}{|c|c|c|c|c|c|}
\hline 14 & $4 \mathrm{~F}(0)$ & 1628 & 2132 & 3332 & $\begin{array}{l}\text { Urea = 8.81 (1H, s,-NH), 7.11-7.61 (4H, m, Ar-H); } \\
\text { Val = 7.72-7.75 }(1 \mathrm{H}, \mathrm{d}, \mathrm{NH}), 4.44-4.52\left(1 \mathrm{H}, \mathrm{t},{ }^{\alpha} \mathrm{CH}\right), 1.86-1.89\left(1 \mathrm{H}, \mathrm{m},-{ }^{-} \mathrm{CH}\right), 1.04-1.09\left(6 \mathrm{H}, \mathrm{d},\left(\mathrm{CH}_{3}\right)_{2}\right) ; \\
\text { Pro }=4.02-4.07\left(1 \mathrm{H}, \mathrm{t},{ }^{\alpha} \mathrm{CH}\right), 1.06-1.31\left(4 \mathrm{H}, \mathrm{m},-{ }^{-\beta}, \mathrm{CH}_{2}\right), 3.40\left(2 \mathrm{H}, \mathrm{t},-\mathrm{CH}_{2}\right)\end{array}$ \\
\hline 15 & $4 \mathrm{~F}(\mathrm{~S})$ & 1618 & 2124 & 3328 & $\begin{array}{l}\text { Thiourea = 8.96 }(1 \mathrm{H}, \mathrm{s},-\mathrm{NH}), 6.84-7.38(4 \mathrm{H}, \mathrm{m}, \mathrm{Ar}-\mathrm{H}) \\
\text { Val = 7.69-7.70 }(1 \mathrm{H}, \mathrm{d}, \mathrm{NH}), 4.33-4.49\left(1 \mathrm{H}, \mathrm{t},-^{\alpha} \mathrm{CH}\right), 1.86-1.92\left(1 \mathrm{H}, \mathrm{m},-{ }^{\beta} \mathrm{CH}\right), 1.03-1.07\left(6 \mathrm{H}, \mathrm{d},\left(\mathrm{CH}_{3}\right)_{2}\right) ; \\
\text { Pro }=3.72-3.80\left(1 \mathrm{H}, \mathrm{t},{ }^{-} \mathrm{CH}\right), 2.97-3.52\left(2 \mathrm{H}, \mathrm{t},{ }^{-} \mathrm{CH}_{2}\right), 1.90-2.91\left(4 \mathrm{H}, \mathrm{m},{ }^{-\beta, \gamma} \mathrm{CH}_{2}\right)\end{array}$ \\
\hline 16 & $3 \mathrm{Cl}(\mathrm{O})$ & 1620 & & 3325 & $\begin{array}{l}\text { Urea = } 8.75(1 \mathrm{H}, \mathrm{s},-\mathrm{NH}), 6.82-7.31(4 \mathrm{H}, \mathrm{m}, \mathrm{Ar}-\mathrm{H}) ; \\
\text { Val = 7.73-7.75 }(1 \mathrm{H}, \mathrm{d}, \mathrm{NH}), 4.34-4.42\left(1 \mathrm{H}, \mathrm{t},{ }^{\alpha} \mathrm{CH}\right), 1.86-1.89\left(1 \mathrm{H}, \mathrm{m},-{ }^{-} \mathrm{CH}\right), 1.04-1.09\left(6 \mathrm{H}, \mathrm{d},\left(\mathrm{CH}_{3}\right)_{2}\right) ; \\
\text { Pro }=3.70-3.79\left(1 \mathrm{H}, \mathrm{t},{ }^{\alpha} \mathrm{CH}\right), 2.95-3.40\left(2 \mathrm{H}, \mathrm{t},{ }^{-} \mathrm{CH}_{2}\right), 1.92-2.91\left(4 \mathrm{H}, \mathrm{m},-\mathrm{\beta}^{-}, \mathrm{CH}_{2}\right)\end{array}$ \\
\hline 17 & $3 \mathrm{Cl}(\mathrm{S})$ & 1624 & 2124 & 3329 & $\begin{array}{l}\text { Thiourea = } 8.98(1 \mathrm{H}, \mathrm{s},-\mathrm{NH}), 6.84-7.22(4 \mathrm{H}, \mathrm{m}, \mathrm{Ar}-\mathrm{H}) ; \\
\text { Val = 7.70-7.72 }(1 \mathrm{H}, \mathrm{d}, \mathrm{NH}), 4.33-4.44\left(1 \mathrm{H}, \mathrm{t},{ }^{\alpha} \mathrm{CH}\right), 1.88-1.90\left(1 \mathrm{H}, \mathrm{m},{ }^{-\beta} \mathrm{CH}\right), 1.03-1.04\left(6 \mathrm{H}, \mathrm{d},\left(\mathrm{CH}_{3}\right)_{2}\right) ; \\
\text { Pro }=3.73-3.83\left(1 \mathrm{H}, \mathrm{t},{ }^{\alpha} \mathrm{CH}\right), 2.97-3.52\left(2 \mathrm{H}, \mathrm{t},{ }^{-} \mathrm{CH}_{2}\right), 1.91-2.95\left(4 \mathrm{H}, \mathrm{m},-^{-\beta, \gamma} \mathrm{CH}_{2}\right)\end{array}$ \\
\hline 18 & $4 \mathrm{Cl}(\mathrm{S})$ & 1620 & 2127 & 3320 & $\begin{array}{l}\text { Thiourea = } 8.94(1 \mathrm{H}, \mathrm{s},-\mathrm{NH}), 6.81-7.32(4 \mathrm{H}, \mathrm{m}, \mathrm{Ar}-\mathrm{H}) ; \\
\text { Val = 7.72-7.75 }(1 \mathrm{H}, \mathrm{d}, \mathrm{NH}), 4.36-4.49\left(1 \mathrm{H}, \mathrm{t},-^{-} \mathrm{CH}\right), 1.85-1.92\left(1 \mathrm{H}, \mathrm{m},{ }^{-\beta} \mathrm{CH}\right), 1.04-1.07\left(6 \mathrm{H}, \mathrm{d},\left(\mathrm{CH}_{3}\right)_{2}\right) ; \\
\text { Pro }=3.72-3.81\left(1 \mathrm{H}, \mathrm{t},{ }^{\alpha} \mathrm{CH}\right), 2.98-3.52\left(2 \mathrm{H}, \mathrm{t},{ }^{-} \mathrm{CH}_{2}\right), 1.93-2.89\left(4 \mathrm{H}, \mathrm{m},{ }^{-\beta}, \mathrm{CH}_{2}\right)\end{array}$ \\
\hline 19 & $3 \mathrm{Br}(0)$ & 1630 & & 3317 & $\begin{array}{l}\text { Urea = 8.78 }(1 \mathrm{H}, \mathrm{s},-\mathrm{NH}), 6.81-7.31(4 \mathrm{H}, \mathrm{m}, \mathrm{Ar}-\mathrm{H}) ; \\
\text { Val = 7.71-7.73 }(1 \mathrm{H}, \mathrm{d}, \mathrm{NH}), 4.34-4.42\left(1 \mathrm{H}, \mathrm{t},{ }^{\alpha} \mathrm{CH}\right), 1.85-1.88\left(1 \mathrm{H}, \mathrm{m},{ }^{-}{ }^{-} \mathrm{CH}\right), 1.04-1.08\left(6 \mathrm{H}, \mathrm{d},\left(\mathrm{CH}_{3}\right)_{2}\right) ; \\
\text { Pro = 3.75-3.85 }\left(1 \mathrm{H}, \mathrm{t},{ }^{-} \mathrm{CH}\right), 2.89-3.40\left(2 \mathrm{H}, \mathrm{t},{ }^{-} \mathrm{CH}_{2}\right), 1.96-2.93\left(4 \mathrm{H}, \mathrm{m},{ }^{-\beta}, \mathrm{CH}_{2}\right)\end{array}$ \\
\hline 20 & $3 \mathrm{Br}(\mathrm{S})$ & 1628 & 2122 & 3320 & $\begin{array}{l}\text { Thiourea = 8.94 }(1 \mathrm{H}, \mathrm{s},-\mathrm{NH}), 6.83-7.32(4 \mathrm{H}, \mathrm{m}, \mathrm{Ar}-\mathrm{H}) ; \\
\text { Val = 7.69-7.71 }(1 \mathrm{H}, \mathrm{d}, \mathrm{NH}), 4.36-4.49\left(1 \mathrm{H}, \mathrm{t},-^{-} \mathrm{CH}\right), 1.86-1.95\left(1 \mathrm{H}, \mathrm{m},{ }^{-\beta} \mathrm{CH}\right), 1.04-1.06\left(6 \mathrm{H}, \mathrm{d},\left(\mathrm{CH}_{3}\right)_{2}\right) ; \\
\text { Pro }=3.71-3.79\left(1 \mathrm{H}, \mathrm{t},{ }^{-} \mathrm{CH}\right), 2.96-3.52\left(2 \mathrm{H}, \mathrm{t},{ }^{-} \mathrm{CH}_{2}\right), 1.88-2.89\left(4 \mathrm{H}, \mathrm{m},{ }^{-\beta}, \mathrm{CH}_{2}\right)\end{array}$ \\
\hline 21 & $3 F(0)$ & 1625 & & 3331 & 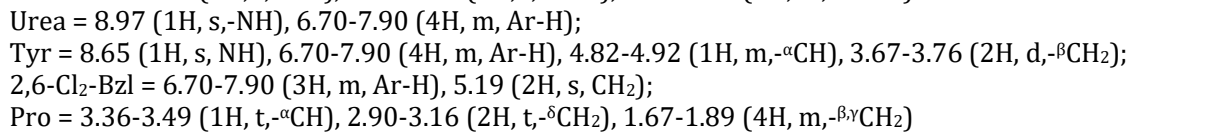 \\
\hline 22 & $4 \mathrm{~F}(0)$ & 1624 & & 3328 & 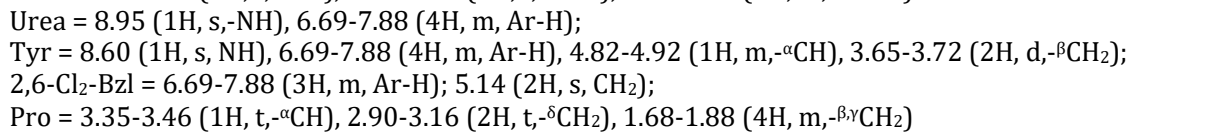 \\
\hline 23 & $4 \mathrm{~F}(\mathrm{~S})$ & 1623 & 2125 & 3322 & 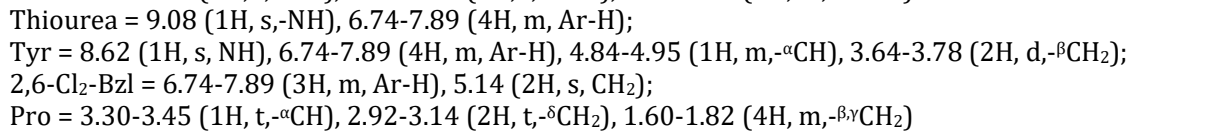 \\
\hline 24 & $3 \mathrm{Cl}(0)$ & 1620 & & 3329 & $\begin{array}{l}\text { Urea = } 8.95(1 \mathrm{H}, \mathrm{s},-\mathrm{NH}), 6.75-7.91(4 \mathrm{H}, \mathrm{m}, \mathrm{Ar}-\mathrm{H}) ; \\
\text { Tyr = } 8.62(1 \mathrm{H}, \mathrm{s}, \mathrm{NH}), 6.75-7.91(4 \mathrm{H}, \mathrm{m}, \mathrm{Ar}-\mathrm{H}), 4.82-4.92\left(1 \mathrm{H}, \mathrm{m},-^{\alpha} \mathrm{CH}\right), 3.68-3.75\left(2 \mathrm{H}, \mathrm{d},-\mathrm{-}^{-} \mathrm{CH}_{2}\right) ; \\
2,6-\mathrm{Cl}_{2}-\mathrm{Bzl}=6.75-7.91(3 \mathrm{H}, \mathrm{m}, \mathrm{Ar}-\mathrm{H}), 5.19\left(2 \mathrm{H}, \mathrm{s}, \mathrm{CH}_{2}\right) ; \\
\text { Pro }=3.33-3.45\left(1 \mathrm{H}, \mathrm{t},{ }^{\alpha} \mathrm{CH}\right), 2.92-3.16\left(2 \mathrm{H}, \mathrm{t},{ }^{-} \mathrm{CH}_{2}\right), 1.64-1.86\left(4 \mathrm{H}, \mathrm{m},-{ }^{-\beta, \gamma} \mathrm{CH}_{2}\right)\end{array}$ \\
\hline 25 & $3 \mathrm{Cl}(\mathrm{S})$ & 1619 & 2125 & 3330 & 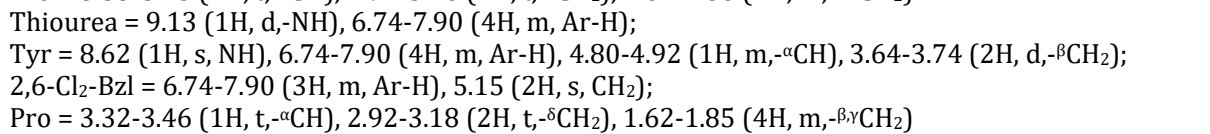 \\
\hline 26 & $4 \mathrm{Cl}(\mathrm{S})$ & 1622 & 2126 & 3328 & 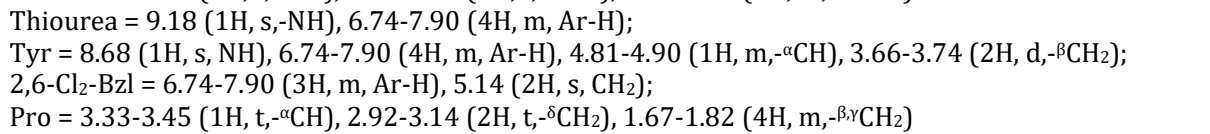 \\
\hline 27 & $3 \mathrm{Br}(0)$ & 1624 & & 3321 & 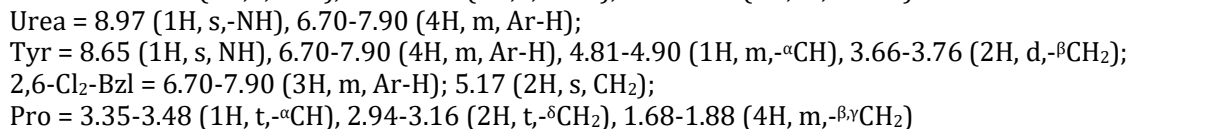 \\
\hline 28 & $3 \mathrm{Br}(\mathrm{S})$ & 1625 & 2130 & 3325 & $\begin{array}{l}\text { Thiourea = } 9.15(1 \mathrm{H}, \mathrm{s},-\mathrm{NH}), 6.71-7.89(4 \mathrm{H}, \mathrm{m}, \mathrm{Ar}-\mathrm{H}) ; \\
\text { Tyr = } 8.63(1 \mathrm{H}, \mathrm{s}, \mathrm{NH}), 6.71-7.89(4 \mathrm{H}, \mathrm{m}, \mathrm{Ar}-\mathrm{H}), 4.82-4.95\left(1 \mathrm{H}, \mathrm{m},{ }^{-\alpha} \mathrm{CH}\right), 3.64-3.74\left(2 \mathrm{H}, \mathrm{d},{ }^{\beta} \mathrm{CH}_{2}\right) ; \\
2,6-\mathrm{Cl}_{2}-\mathrm{Bzl}=6.71-7.89(3 \mathrm{H}, \mathrm{m}, \mathrm{Ar}-\mathrm{H}), 5.15\left(2 \mathrm{H}, \mathrm{s}, \mathrm{CH}_{2}\right) ; \\
\text { Pro }=3.34-3.47\left(1 \mathrm{H}, \mathrm{t},{ }^{\alpha} \mathrm{CH}\right), 2.92-3.14\left(2 \mathrm{H}, \mathrm{t},{ }^{-} \mathrm{CH}_{2}\right), 1.60-1.82\left(4 \mathrm{H}, \mathrm{m},-{ }^{-\beta, \gamma C \mathrm{CH}_{2}}\right)\end{array}$ \\
\hline 29 & $3 F(0)$ & 1617 & & 3328 & 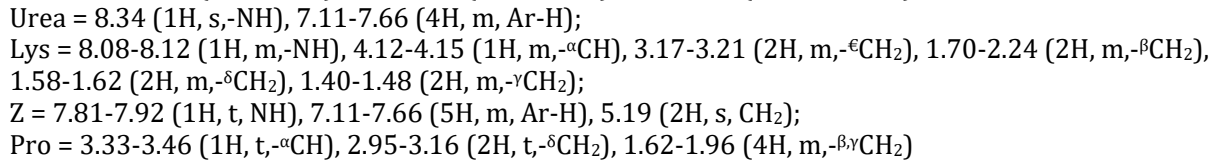 \\
\hline 30 & $4 \mathrm{~F}(0)$ & 1628 & & 3331 & 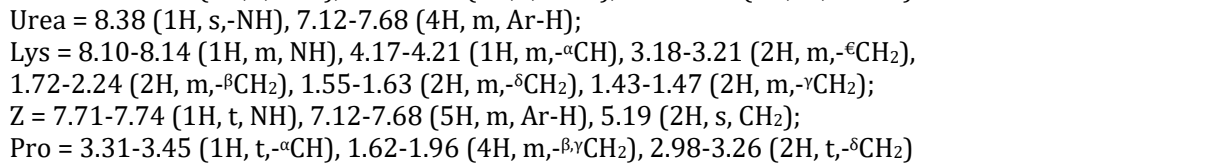 \\
\hline 31 & $4 \mathrm{~F}(\mathrm{~S})$ & 1624 & 2130 & 3327 & 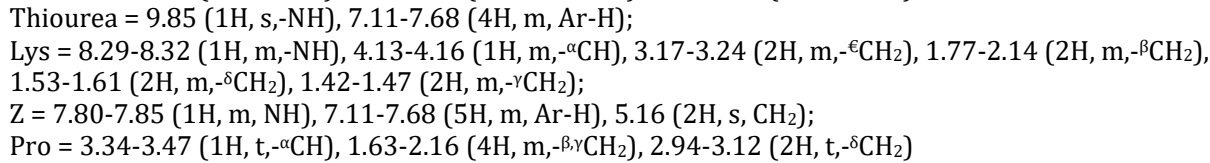 \\
\hline 32 & $3 \mathrm{Cl}(0)$ & 1626 & & 3328 & 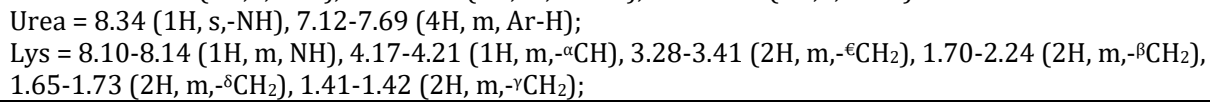 \\
\hline
\end{tabular}




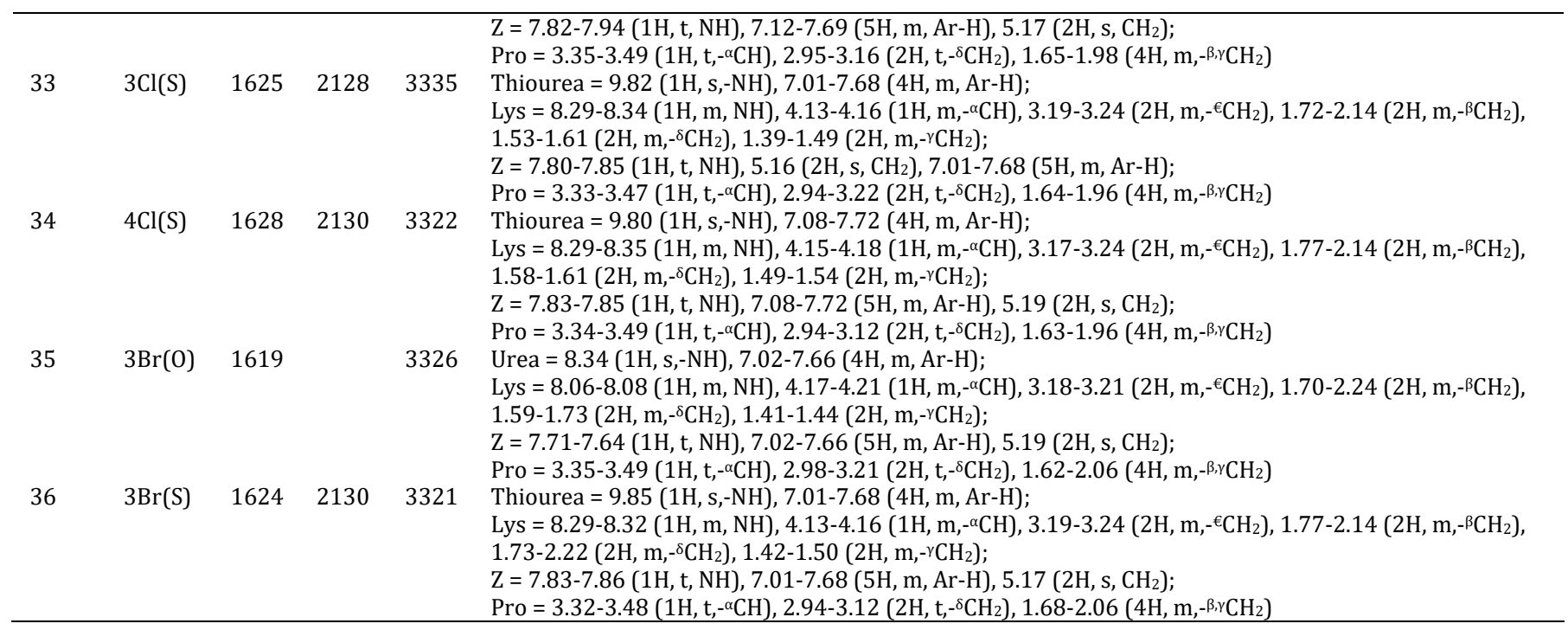

IR = Infrared spectroscopic values, NMR = Nuclear Magnetic Resonance spectroscopic values, DMSO = Dimethyl sulfoxide.

Table 3: Antiurease studies of the synthesized compounds (1-36). Each value represents mean \pm SD $(n=3)$

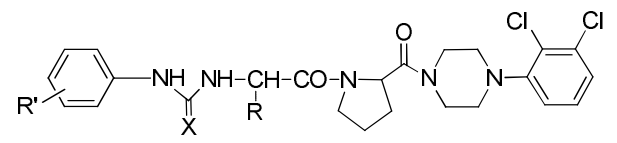

$\mathrm{R}^{\prime}=\mathrm{F}, \mathrm{Cl}, \mathrm{Br} ; \mathrm{R}=$ Side chain of amino acids; $\mathrm{X}=\mathrm{O} / \mathrm{S}$

\begin{tabular}{|c|c|c|c|}
\hline Entry & $\mathbf{R}$ & $\mathbf{X}$ & Antiurease activity $(\mu \mathrm{M})^{a}$ \\
\hline 1 & Boc-FP-PZN & & $78.5 \pm 0.69$ \\
\hline 2 & Boc-VP-PZN & & $92.1 \pm 0.55$ \\
\hline 3 & Boc-Y(2,6CL2Bzl)P-PZN & & $56.7 \pm 0.55$ \\
\hline 4 & Boc-K(Z)P-PZN & & $32.5 \pm 0.92$ \\
\hline 5 & $3 F$ & 0 & $23.5 \pm 0.18$ \\
\hline 6 & $4 \mathrm{~F}$ & 0 & $15.8 \pm 0.13$ \\
\hline 7 & $4 \mathrm{~F}$ & $\mathrm{~S}$ & $6.0 \pm 0.11$ \\
\hline 8 & $3 \mathrm{Cl}$ & 0 & $20.7 \pm 0.25$ \\
\hline 9 & $3 \mathrm{Cl}$ & $\mathrm{S}$ & $18.2 \pm 0.31$ \\
\hline 10 & $4 \mathrm{Cl}$ & $\mathrm{S}$ & $15.0 \pm 0.15$ \\
\hline 11 & $3 \mathrm{Br}$ & 0 & $28.0 \pm 0.12$ \\
\hline 12 & $3 \mathrm{Br}$ & $\mathrm{S}$ & $25.7 \pm 0.47$ \\
\hline 13 & $3 \mathrm{~F}$ & 0 & $28.0 \pm 0.59$ \\
\hline 14 & $4 \mathrm{~F}$ & 0 & $21.6 \pm 0.24$ \\
\hline 15 & $4 \mathrm{~F}$ & $\mathrm{~S}$ & $7.4 \pm 0.18$ \\
\hline 16 & $3 \mathrm{Cl}$ & 0 & $31.8 \pm 0.64$ \\
\hline 17 & $3 \mathrm{Cl}$ & $\mathrm{S}$ & $30.6 \pm 0.96$ \\
\hline 18 & $4 \mathrm{Cl}$ & $\mathrm{S}$ & $21.1 \pm 0.21$ \\
\hline 19 & $3 \mathrm{Br}$ & 0 & $45.6 \pm 0.95$ \\
\hline 20 & $3 \mathrm{Br}$ & $\mathrm{S}$ & $32.5 \pm 0.33$ \\
\hline 21 & $3 \mathrm{~F}$ & 0 & $20.0 \pm 0.25$ \\
\hline 22 & $4 \mathrm{~F}$ & 0 & $12.0 \pm 0.36$ \\
\hline 23 & $4 \mathrm{~F}$ & $\mathrm{~S}$ & $2.0 \pm 0.12$ \\
\hline 24 & $3 \mathrm{Cl}$ & 0 & $7.0 \pm 0.21$ \\
\hline 25 & $3 \mathrm{Cl}$ & $\mathrm{S}$ & $4.4 \pm 0.18$ \\
\hline 26 & $4 \mathrm{Cl}$ & $\mathrm{S}$ & $3.0 \pm 0.14$ \\
\hline 27 & $3 \mathrm{Br}$ & 0 & $18.3 \pm 0.25$ \\
\hline 28 & $3 \mathrm{Br}$ & $\mathrm{S}$ & $16.0 \pm 0.26$ \\
\hline 29 & $3 \mathrm{~F}$ & 0 & $27.2 \pm 0.76$ \\
\hline 30 & $4 \mathrm{~F}$ & 0 & $18.4 \pm 0.41$ \\
\hline 31 & $4 \mathrm{~F}$ & $\mathrm{~S}$ & $3.12 \pm 0.08$ \\
\hline 32 & $3 \mathrm{Cl}$ & 0 & $21.8 \pm 0.72$ \\
\hline 33 & $3 \mathrm{Cl}$ & $\mathrm{S}$ & $19.2 \pm 0.32$ \\
\hline 34 & $4 \mathrm{Cl}$ & $\mathrm{S}$ & $8.91 \pm 0.17$ \\
\hline 35 & $3 \mathrm{Br}$ & 0 & $28.4 \pm 0.41$ \\
\hline 36 & $3 \mathrm{Br}$ & $\mathrm{S}$ & $21.0 \pm 0.22$ \\
\hline Thiourea & & & $21.0 \pm 0.11$ \\
\hline
\end{tabular}

avalues are mean of three determinations, the range of which are $<5 \%$ of mean in all cases 


\section{Pharmacology}

The focus of our approach in this work was to evaluate the urease inhibitory activity of the title compounds by in vitro method. Data obtained is presented in table 3. Thiourea was used as the reference standard in the assay. Analysis of the data from this study suggests that (1) Most of the synthesized urea and thiourea derivatives with substitution on the phenyl ring were found to be more active than standard thiourea, (2) the activity of the synthesized compounds is dependent upon the nature of amino acids which is bridging the urea/thiourea group and the heterocycle and also position of the substitution on the aromatic ring of urea/thioureas.

Among the four series of dipeptides, Tyr containing dipeptide conjugated heterocyclic derivatives (23-26) were found to be most active followed by Lys dipeptide conjugated heterocyclic compounds (29-36). On the other hand, Phe dipeptide conjugated heterocyclic compounds (5-12) and Val dipeptide conjugated heterocyclic compounds (13-20) have shown moderate activity. The observed results revealed that substitution pattern on the phenyl ring of ureas/thioureas plays very important role on the activity. Compounds which have thiourea group 7, 10, 15, 23, 25, 26 and 31 have exhibited high potent activity with an $\mathrm{IC}_{50}$ values of $10.0 \pm 0.11$, $15.0 \pm 0.15,17.4 \pm 0.18,4.0 \pm 0.12,7.0 \pm 0.21,4.4 \pm 0.18,2.0 \pm 0.14$ and $3.12 \pm 0.08 \mu \mathrm{M}$ respectively. The remarkable activity of compounds $23,25,26$ and 31 may be due to electron withdrawing substituent which is more desirable. Further, it was noticed that changing in substitution position on the aryl ring changes the activity. When substituents present at para and meta position exhibited maximum activity compared to ortho. Compounds containing thiourea have emerged as active moieties which indicated that involving ' $\mathrm{S}$ ' in the design would be more beneficial which could attribute to the electronegative behaviour of ' $\mathrm{S}$ ' and its ability to react with target group there by increasing the efficiency of the compounds.

The urease inhibition capacity of newly synthesized molecules was compared with the literature reported other studies. Molecules like morpholine [4], metronidazole [6], substituted piperidines [7], Barbiturates [8] and benzoquinone derivatives [9] having $\mathrm{IC}_{50}$ values $2.37 \mu \mathrm{M}, 12 \mu \mathrm{M}, 13 \mu \mathrm{M}, 19.38 \mu \mathrm{M}$ and $27.30 \mu \mathrm{M}$ respectively. Most of our analogues were found to be more active than the results with those of other studies. Importantly the analogue 23 was nearly 10 fold $(2 \mu \mathrm{M})$ more potent than the reference standard, $21.0 \pm 0.11 \mu \mathrm{M}$.

We were interested to give a preliminary mechanistic approach for the ability of the synthesized compounds to inhibit the urease activity i.e., the inhibition of the production of ammonia. Urease is a dinickel containing enzyme surrounded by molecules. In literature, it has been described that urea binds to nickle using the carbonyl oxygen and displaces the water molecules. This further facilitates the urea coordination to the second $\mathrm{Ni}^{2+}$ by hydrogen binding. Nucleophilic attack of oxygen (of $\mathrm{OH}$ ) to carbonyl carbon followed by proton abstraction leads to the elimination of ammonia molecule [29]. More the concentration of ammonia, lesser will be the activity. Thus in the present work substituted urea and thiourea derivatives would not result in the production of ammonia and hence it may be concluded that compounds of the type described in this work may act as more active analogues.

\section{CONCLUSION}

In conclusion, four series of dipeptides [FP, VP, Y(2,6-Cl $-\mathrm{Bzl}) \mathrm{P}$ and $\mathrm{K}(\mathrm{Z}) \mathrm{P}]$ conjugated 2,3-dichlorophenyl piperazine were synthesized and converted into urea and thiourea derivatives by reacting with various substituted phenyl isocyanates/isothiocyanates. All the synthesized compounds were evaluated for their urease inhibitory potential. These inhibitors demonstrated significant in vitro efficacy as antifreeze agents. Compounds with ' $\mathrm{S}$ ' and preferably $\mathrm{F}$ and $\mathrm{Cl}$ substituents at meta and para positions showed predominant urease inhibitory activity. These results suggest the suitability of the designed molecular frame work for use in the development of anti-urease therapy.

\section{ACKNOWLEDGEMENT}

The authors gratefully acknowledge University Grants Commission (UGC), New Delhi for awarding UGC-Post Doctoral Fellowship (PDFSS), BSR faculty fellowship and DST Inspire fellowship.

\section{AUTHORS CONTRIBUTION}

D. M. Suyoga Vardhan and D. Channe Gowda involved in the synthesis of urea and thiourea derivatives of dipeptides conjugated 2,3-dichlorophenyl piperazine and their characterization. H. K. Kumara and H. Pavan Kumar contributed in urease inhibition assay.

\section{CONFLICT OF INTERESTS}

The authors declare that they have no conflict of interest

\section{REFERENCES}

1. Mobley HLT, Island MD, Hausinger RP. Molecular biology of microbial ureases. Microbiol Rev 1995;59:451-80.

2. Mobley HLT, Island MD, Hausinger RP. Microbial ureases: significance, regulation and molecular characterization. Microbiol Rev 1989;53:85-102.

3. Samtoy, DeBeukelaer BM. Ammonia encephalopathy secondary to urinary tract infection with proteuis mirabilis. Pediatrics 1980;65:294-7.

4. Betkas H, Ceylan S, Demisbas N, Alpay-Karaoglu S, Sokmen BB. Antimicrobial and anti-urease activities of newly synthesized morpholine derivatives containing an azole nucleus. Med Chem Res 2013;22:3629-39.

5. Khan I, Ali S, Hameed S, Rama NH, Hussain MT, Wadood A, et al. Synthesis, antioxidant activities and urease inhibition of some new 1,2,4-triazole and 1,3,4-thiadiazole derivatives. Eur J Med Chem 2010;45:5200-7.

6. Mao WJ, Lv PC, Shi L, Lee HQ, Zhu HL. Synthesis, molecular docking and biological evaluation of metronidazole derivatives as potent Helicobacter pylori urease inhibitors. Bioorg Med Chem 2009;17:7531-6.

7. Khan KM, Saify ZS, Lodhi MA, Buttz N, Perveen S, Maharvi GM, et al. Piperidines: a new class of urease inhibitors. Nat Prod Res 2006;20:523-8.

8. Khan KM, Ali M, Wadood A, Zaheer Ul Haq, Khan M, Lodhi MA, et al. Molecular modeling-based antioxidant arylidene barbiturates as urease inhibitors. J Mol Graphics Modell 2011;30:153-6.

9. You ZL, Xian DM, Zhang M, Cheng XS, Li XF. Synthesis, biological evaluation and molecular docking studies of 2,5-substituted1,4-benzoquinone as novel urease inhibitors. Bioorg Med Chem 2012;20:4889-94.

10. Upadhyaya RS, Kulkarni GM, Vasireddy NR, Vandavasi JK, Dixit SS, Sharma V, et al. Design, synthesis and biological evaluation of novel triazole, urea and thiourea derivatives of quinoline against Mycobacterium tuberculosis. Bioorg Med Chem 2009;17:4681-92.

11. Suresha GP, Suhas R, Kapfo W, Gowda DC. Urea/thiourea derivatives of quinazolinone lysine conjugates: synthesis and structure-activity relationships of a new series of antimicrobials. Eur J Med Chem 2011;46:2530-40.

12. Faidallah HM, Khan KA, Asiri AM. Synthesis and biological evaluation of new 3,5-di(trifluoromethyl)-1,2,4-triazolesulfonylurea and thiourea derivatives as antidiabetic and antimicrobial agents. J Fluorine Chem 2011;132:870-7.

13. Venkatachalam TK, Maob C, Uckun FM. Effect of stereochemistry on the anti-HIV activity of chiral thiourea compounds. Bioorg Med Chem 2004;12:4275-84.

14. Sunduru ZN, Srivastava K, Rajakumar S, Ouri SK, Saxena JK, Chauhan PMS. Synthesis of novel thiourea, thiazolidinedione and thioparabanic acid derivatives of 4-aminoquinoline as potent antimalarials. Bioorg Med Chem Lett 2009;19:2570-3.

15. Zhi-Jian C, Ya Na C, Chun $\mathrm{Na} \mathrm{Xu,} \mathrm{Shan-Shan} \mathrm{Zhao,} \mathrm{Qui-Yue} \mathrm{Cao,}$ Shao-Sang Qian, et al. Synthesis, crystal structures, molecular docking, and in vitro biological activities evaluation of transition metal complexes with 4-(3,4-dichlorophenyl) piperazine-1-carboxylic acid. J Mol Struct 2016;1117:293-9.

16. ABD-El-Aziz AS, Abdelghani AA, El-Sadany SK, Overy DP, Kerr RG. Antimicrobial and anticancer activities of organoiron melamine dendrimers capped with piperazine moieties. Eur Polym J 2016;82:307-23.

17. Silva DPB, Florentino IF, Oliveria LP, Lino RC, Galdino PM, Menegetti $\mathrm{R}$, et al. Antinociceptive and anti-inflammatory activities of 4-[(1-phenyl-1H-pyrazol-4-yl) methyl] 1- 
piperazine carboxylic acid ethyl ester: a new piperazine derivative. Pharmacol Biochem Behav 2015;137:86-92.

18. Wynn RL. New antiplatelet and anticoagulant drugs. Gen Dent 2012;60:8-11.

19. Barman A, Shivaprakash S, Latha D, Reddy GC. Acute toxicity study of (z)-1-benzhydryl-4-cinnamylpiperazines in swiss albino mice. Asian J Pharm Clin Res 2015;8:223-4.

20. Suresha GP, Suhas R, Wethroe K, Gowda DC. Urea/thiourea derivatives derivatives of quinazolinone-lysine conjugates: synthesis and structure activity relationships of a new series of antimicrobials. Eur J Med Chem 2011;46:2530-40.

21. Shantharam CS, Suyoga Vardhan DM, Suhas R, Sridhara MB, Channe Gowda D. Inhibition of protein glycation by urea and thiourea derivatives of glycine/proline conjugated benzisoxazole analogue-synthesis and structure activity studies. Eur J Med Chem 2013;60:325-32.

22. Suyoga Vardhan DM, Shantharam CS, Suhas R, Sridhra MB, Channe Gowda D. Synthesis and SAR studies of urea and thiourea derivatives of Gly/Pro conjugated to piperazine analogue as potential AGE inhibitors. Protein Peptde Lett 2013;20:888-97.

23. Suhas R, Chandrashekar S, Gowda DC. Synthesis of ureido and thioureido derivatives of peptides conjugated heterocycles-a new class of promising antimicrobials. Eur J Med Chem 2012;48:179-91.
24. Gowda BKK, Prasad HS, Rangappa KS, Gowda DC. Hydrophobicity dependence of oxidation of tetrapeptides of elastin sequences with Mn(III): synthesis, characterization, kinetics and mechanistic study. Int J Chem Kinet 2002;34:39-48.

25. Prasad KU, Iqbal MA, Urry DW. Utilization of 1-hydroxy benzotriazole in mixed anhydride coupling reactions. Int J Pept Protein Res 1985;25:408-13.

26. Y Oshiro, S Tokushima, Sata and Itana. U. S. Patent; 1991;5,006,528.

27. Weatherburn MW. Phenol-hypochlorite reaction for determination of ammonia. Anal Chem 1967;39:971-4.

28. Khan I, Ali S, Hamed S, Rama NH, Hussain MT, Wadood A, et al. Synthesis, antioxidant activities and urease inhibition of some new 1,2,4-triazole and 1,3,4-thiadiazole derivatives. Eur J Med Chem 2010;45:5200-7.

29. Zambelli B, Musiani F, Benini S, Ciurli S. Chemistry of $\mathrm{Ni}^{2+} \mathrm{in}$ urease: sensing, trafficking and catalysis. Acc Chem Res 2011;44:520-30.

\section{How to cite this article}

- DM Suyoga Vardhan, HK Kumara, H Pavan Kumar, D Channe Gowda. Inhibition of urease enzyme activity by urea and thiourea derivatives of dipeptides conjugated 2,3-dichlorophenyl piperazine. Int J Pharm Pharm Sci 2017;9(9):92-99. 